\title{
PERSPECTIVE
}

Cite this: DOI: 10.1039/x0xx00000x

\section{Prospects about Magnetic Nanoparticles for Systemic Administration: Synthesis and Quantitative Detection}

\author{
L. Gutiérrez ${ }^{a}$, M. P. Morales ${ }^{a}$ and F.J. Lázaro ${ }^{b}$
}

Received 00th January 2012,

Accepted 00th January 2012

DOI: $10.1039 / \times 0 \times x 00000 x$

www.rsc.org/

\begin{abstract}
Methods for the quantitative determination of magnetic nanoparticles in biological matrices, in the frame of biomedical applications, are required to evaluate the particles biodistribution after systemic administration. AC magnetic susceptibility measurements are an alternative method to quantify magnetic nanoparticles in tissues, being able to provide also information on the particle transformations over time and allowing the distinction of the particles from other endogenous species such as the ferritin iron cores. The protocol for particle quantification using AC magnetic susceptibility measurements is described in detail in this article. A summary of magnetic nanoparticles synthesis routes is also provided.
\end{abstract}

\section{Introduction}

Over the past decades a surge of studies about nanotechnology has appeared ${ }^{1}$. Some of the most studied materials for nanotechnology applications are magnetic nanoparticles. More specifically, the range of applications in which magnetic nanoparticles are used is becoming more diverse over the years, including topics so different as water pollution treatments ${ }^{2}$ or parasite diagnostics techniques ${ }^{3}$. Medical applications, from diagnosis to treatment, of magnetic nanoparticles are also being widely studied. In particular, magnetic nanoparticles are being used as contrast agents for magnetic resonance imaging, magnetic carriers for drug delivery or heating sources for hyperthermia in cancer treatment ${ }^{4,5}$.

The research community is making a big effort to improve the particles properties, such as the particle size, shape, charge, magnetic behaviour or heating characteristics, depending on the application ${ }^{6}$. Nonetheless, after finding the appropriated materials there are still a few challenges to be solved before the research work is transformed into commercially viable medical products ${ }^{7}$. Once in vitro studies have been performed in cell cultures to assure the efficacy and non-toxicity of the particles ${ }^{8}$, animal models are used to study the pharmacokinetics of the particles. In such cases, it is crucial to be able to determine first the magnetic nanoparticles biodistribution following systemic administration. Then, it is also necessary to evaluate the transformations and fate of the particles to ensure safety. An example of the great importance of the study of the risks associated to nanoparticles ${ }^{9}$ are the programs initiated by the Unites States, Europe or Japan on the safety evaluation of nanomaterials ${ }^{10}$.

Detecting and quantifying magnetic nanoparticles in a biological matrix, is not a simple task. Several techniques are being used to quantify the amount of magnetic nanoparticles within tissues; however, none of them is established as a golden standard for the particle analysis within a tissue yet, as all of them present some drawbacks. The available characterization techniques fail to provide quantitative data, ultrastructural location of the particles or information of the physicochemical transformations of the particles at the same time. Therefore combinations of several techniques are regularly used to analyse the fate and transformation of the particles within cells or tissues ${ }^{11}$.

Non-invasive quantitative approaches to analyse magnetic nanoparticles within tissues include measurement of tissue $R_{2}$ changes by magnetic resonance imaging (MRI) ${ }^{12,} 13$ and radionuclide labelling of the nanoparticles with further detection by positron emission tomography (PET) or singlephoton emission computed tomography (SPECT) ${ }^{14}$. Radiation safety issues are one of the major drawbacks for PET and SPECT measurements. Furthermore, as the addition of the radioisotope may affect the physicochemical properties of the nanoparticles, it remains unproven that the non-labelled nanoparticles have the same biodistribution as the labelled ones.

To get a deep understanding of the problem, ex vivo studies of tissue samples are currently performed by using a wide range of characterisation techniques, many of them of common use in Materials Physics and Chemistry. Transmission electron microscopy (TEM) provides useful ultrastructural information on the location and aggregation degree of the particles in the tissue, and informs about possible degradation processes ${ }^{15-17}$. However, hindered by the low dosage of particles rationally used in biomedicine, their detection by electron microscopy may sometimes be equivalent to looking for a needle in a 
haystack. Elemental analyses, through either optical or mass spectroscopies, are very sensitive ways to detect certain metals that may belong to the composition of the magnetic nanoparticles. Nevertheless, as to limit toxicity most of the magnetic nanoparticles for biomedical applications are ironbased, the quantification of this metal, chosen to be a surrogate for the presence of nanoparticles, is interfered by the endogenous iron. Other techniques used for the analysis of magnetic nanoparticles either in cells or tissues are Electron Spin Resonance $(\mathrm{ESR})^{12}$, Ferromagnetic Resonance ${ }^{18}$ and ultrasounds ${ }^{19}$.

The magnetic properties of nanoparticles have also been involved in other quantification approaches. More specifically, the resonant behaviour of the particles magnetic moment ${ }^{20}$ and the time $^{21}$ and field ${ }^{22}$ dependence of the sample magnetic moment have been taken into account. In some cases it is not necessary to bring the sample to low temperatures, allowing for virtual non-invasive quantification. The drawback in some cases is that the success of the method relies on using nanoparticles of uniform magnetic properties as different individual magnetic behaviours may be confused with a different particle concentration. In this context, the use of alternating current (AC) magnetic susceptibility measurements although only applicable to samples brought to low temperatures allows both to discern among different nanoparticle sizes with a virtual quantification of each of them $^{23}$. Moreover, this technique also allows the evaluation of the nanoparticle transformations over time ${ }^{24}$ and the distinction of tissue endogenous iron from magnetic nanoparticles ${ }^{23}$.

In this work, we will describe the quantification protocol to determine the amount of magnetic nanoparticles in tissues based on AC magnetic susceptibility measurements ${ }^{23,25}$. Last advances in synthesis approaches to produce magnetic nanocrystals with uniform shapes and sizes for biomedical applications are also summarized.

\section{Magnetic AC susceptibility}

\section{The experimental technique}

Determination of the AC susceptibility is based on measuring the time dependent magnetic moment that results after applying an alternating magnetic field to the sample. From this experiment, and knowing the sample mass, the so-called inphase $\left(\chi^{\prime}\right)$ and out-of-phase ( $\left.\chi^{\prime \prime}\right)$ components of the AC mass susceptibility are determined. In practice it is possible to apply an AC magnetic field $\mathrm{h}_{\mathrm{AC}}=\mathrm{h}_{0} \cos (\omega \mathrm{t})$, with control on the amplitude $h_{0}$ and the frequency $\omega$. To this end $h_{0}$ is chosen from a compromise between having enough sensitivity and avoiding nonlinear effects, while $\omega$ is taken in the range where the equipment has the best sensitivity (for SQUID detectors values of the order of $10 \mathrm{~Hz}$ are usually a good choice). The measured magnetic moment results to be $\mathrm{m}_{\mathrm{AC}}=\mathrm{m}_{0} \cos (\omega \mathrm{t}-\phi)$, that is, a quantity that varies in time with the same frequency $\omega$ but is delayed with a phase shift $\phi$ with respect to the exciting field. Technically, to describe this time-dependent behaviour, a complex notation is used, where the magnetic mass susceptibility, $\chi=\chi^{\prime}-\mathrm{i} \chi^{\prime \prime}$, is defined in such a way that $\mathrm{M}=\rho \chi$ $\mathrm{H}$, being $\mathrm{M}$ the magnetic moment per unit volume, $\rho$ the sample density and $\mathrm{H}$ the applied magnetic field. Corresponding units in the SI system are $\mathrm{A} / \mathrm{m}, \mathrm{kg} / \mathrm{m}^{3}, \mathrm{~m}^{3} / \mathrm{kg}$ and $\mathrm{A} / \mathrm{m}$ for $\mathrm{M}, \rho, \chi$ and $\mathrm{H}$ respectively.

The measurement of the AC susceptibility was early employed in the characterisation of fine magnetic particles ${ }^{26}$, years before the good control of the synthesis procedures improved their systemic administration in a safe manner. The AC susceptibility of fine particle systems, as a function of amplitude, frequency and temperature, that results from the magnetic moment and magnetic anisotropy of the particles has extensively been studied so far both experimentally and theoretically ${ }^{27-30}$.
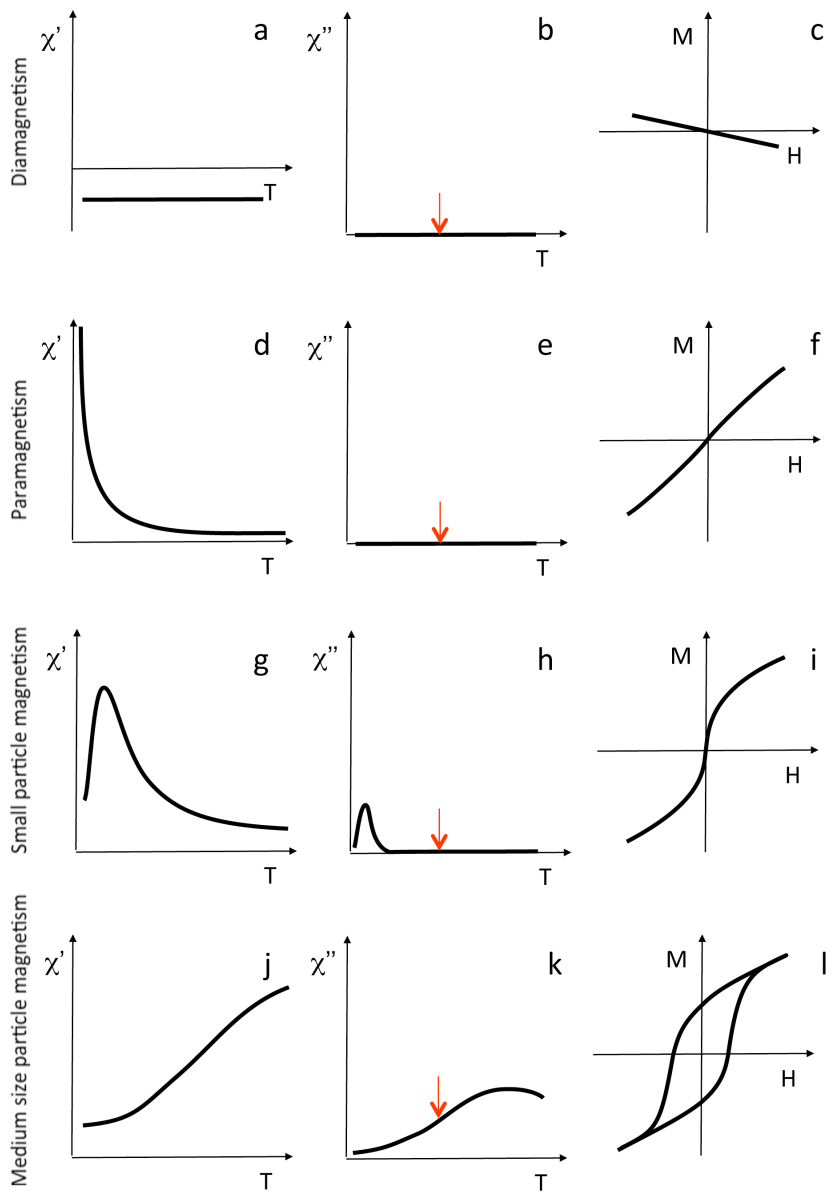

Fig. 1. Temperature dependence of the in-phase susceptibility, $\chi^{\prime}(T)$, (first column), temperature dependence of the out-of-phase susceptibility, $\chi^{\prime \prime}(T)$, (second column) and field dependence of the magnetisation, $M(H)$, (third column) of the typical magnetic species in biological tissues. First raw: diamagnetic species as, e.g., water and many organic molecules. Second raw: metal-containing paramagnetic molecules as, e.g., deoxyhaemoglobin. Third raw: very small mineral magnetic particles, thus superparamagnetic above relatively low temperature, as, e.g., the iron-containing ferritin cores. Fourth raw: larger mineral magnetic particles that become magnetically blocked at relatively high temperature as, e.g., some particulate magnetic carriers. The third column $\mathrm{M}(\mathrm{H})$ data are supposed to be measured at the temperatures indicated by arrows in the second column; for a given temperature, the nonzero out-ofphase susceptibility and the $M(H)$ hysteresis both reveal the difficulties of the particles magnetic moment to follow the $A C$ magnetic field variations. 


\section{Magnetic properties of biological tissues}

The determination of the concentration of administered magnetic particles in tissues by measuring the magnetic AC susceptibility, in the form that is described here, corresponds to analysing tissue samples obtained from biopsy or, in animal model research, from dissection. This approach differs from other non-invasive techniques ${ }^{31,} 32$ in that the analysis is restricted to small samples, typically in the range of 10-100 mg dry weight. The use of small samples, though, makes it possible to get a more accurate understanding of what is actually occurring in the tissue, obtaining information of phenomena like nanoparticle metabolism and aggregation. Eventually, nanoparticle biodistribution studies made through magnetismbased tomographic techniques ${ }^{33}$ will undoubtedly be benefited from the deep understanding of the magnetic properties of these nanoparticles in the tissues.

Samples analysed so far typically meet the requirements of widely disposed magnetic detection equipment that uses SQUID sensors $\uparrow$. Namely, it is possible to characterise both fresh and dried tissues, although much precautions are needed in the former case (maintenance of frozen state) with no significant advantages in the results.

Small organs, such as the spleen or the kidneys, from mouse models may be measured as a whole. However, when dealing with bigger organs (e.g. liver) or bigger animals (e.g. rats, rabbits, etc), it has to be taken into account that a heterogeneous distribution of the particles may exist within each organ ${ }^{34}$. In such cases tissues should either be homogeneized to get an average result of the amount of particles in the tissue, or several tissue portions have to be characterised to evaluate the distribution within the organ.

As far as we shall consider magnetic nanoparticles in a biological tissue it is preceptive to know not only their specific magnetic behaviour but also the magnetic properties of the tissue itself. Biological tissues contain a wide variety of organic compounds together with a variable content of water. All these species as a whole exhibit a negative and temperature independent in-phase magnetic susceptibility (Fig. 1a), a zero out-of-phase component (Fig. 1b), and a negative-slope straight line $\mathrm{M}(\mathrm{H})$ (Fig. 1c) due to the magnetic phenomenon called diamagnetism. In practice, this rather simple magnetic behaviour is easy to subtract from other contributions. In addition to this, some organic molecules do contain transition metal ions as in the case of haemoproteins. It is known in particular that haemoglobin behaves in a diamagnetic way in the oxy- state while it shows paramagnetism in the deoxystate $^{35}$. Paramagnetism is known to exhibit an in-phase magnetic susceptibility of the type $\chi^{\prime}=\mathrm{C} / \mathrm{T}$ (Fig. $1 \mathrm{~d}$ ), where $\mathrm{C}$ is a constant and $\mathrm{T}$ the absolute temperature, together with a zero out-of-phase component $\left(\chi^{\prime \prime}=0\right)$ (Fig. 1e), and a Brillouin law $\mathrm{M}(\mathrm{H})$ behaviour with no hysteresis (Fig. 1f) ${ }^{36}$.

Eventual iron mineralisation in biological tissues takes place in the form of nanometric particles of inorganic iron compounds, mostly iron (oxyhydr-)oxides. Due to their small size, those particles contain just a single magnetic domain and are usually superparamagnetic at room temperature. To this class belong the cores of the iron storage protein ferritin ${ }^{37}$, nanoparticles in iron supplement drugs for intravenous administration ${ }^{38}$ as well as any magnetic carrier of biomedical interest as contrast agents in Magnetic Resonance Imaging $^{39}$, drug delivery ${ }^{40}$ and hyperthermia $^{41}$. Due to their magnetic anisotropy and as temperature decreases, the net magnetic moment of these nanoparticles is not able to follow the inversions imposed by the alternating (AC) exciting magnetic field, giving rise to a magnetic moment $\mathrm{m}_{\mathrm{AC}}$ that evolves in a delayed form with respect to $\mathrm{h}_{\mathrm{AC}}$, and therefore to a nonzero out-of-phase component of the susceptibility $(\chi=0)$ (Fig. 1h, 1k). This phenomenon is progressive as temperature decreases but since it takes place for each nanoparticle in a narrow temperature range it is termed magnetic blocking, being the so called blocking temperature something that one could define for each nanoparticle $^{42}$. In practice, and in biological tissues in particular, the size and nature of the nanoparticles is a distributed parameter. As the magnetic anisotropy, and therefore the blocking temperature, depends on these factors, a nonzero $\chi^{\prime \prime}$ is spread in temperature according to the width of such distribution. These phenomena do also have their consequences on the field dependence of the magnetisation. Namely, $\mathrm{M}(\mathrm{H})$ for non-blocked particles follows a qualitatively similar analog to the Brillouin law (here called Langevin law) (Fig. 1i). On the contrary, when the particles are blocked, $\mathrm{M}(\mathrm{H})$ exhibits a hysteretic behaviour (Fig. 11). It should not be forgotten that, as in this context the particles are single domain, hysteresis is predominantly field sweep speed dependent; this phenomenon should not be confused with magnetic domain motion occurring in the case of larger and massive (multidomain) specimens.

\section{Particulate magnetic species: relevance of the out-of-phase susceptibility}

In a real biological sample, as all the above mentioned magnetic species may be present, its magnetic behaviour will result from the sum of the above described contributions. Nevertheless, it occurs that the only species that significantly contribute to $\chi^{\prime \prime}$ in some temperature range are the magnetic particles (see Figs. 1h, $1 \mathrm{k})$, therefore, the consideration of the $\chi^{\prime \prime}(\mathrm{T})$ profile becomes especially useful to study iron biomineralisation in tissues.

More specifically, $\chi^{\prime \prime}(\mathrm{T})$ is a manifestation of the distribution of the relaxation times needed by the particles magnetic moments to be oriented in space following the time variations of $h_{A C}$. Quantitatively, the expression that describes the out-of-phase mass susceptibility, for experimental frequencies lower than those of ferromagnetic resonance, and for $\mathrm{E}_{\mathrm{a}} /(\mathrm{kT})>>1$, is 27,42

$$
\chi^{\prime \prime}(T)=\frac{\mu_{0}}{3 k T} \int_{0}^{\infty} d D \cdot n(D) \cdot m^{2}(D)\left[\frac{\omega \tau}{\left(1+\omega^{2} \tau^{2}\right)}\right],
$$

where $\tau=\tau_{0} \exp \left(\mathrm{E}_{\mathrm{a}} / \mathrm{kT}\right)$ is the particle relaxation time, $\tau_{0}$ a preexponential factor, typically of the order of $10^{-12} \mathrm{~s}$ for noninteracting magnetic particles, $\mathrm{E}_{\mathrm{a}}$ the single particle 
anisotropy energy $\left(\mathrm{E}_{\mathrm{a}}=\mathrm{K}_{\mathrm{eff}} \mathrm{V}\right)$, where $\mathrm{K}_{\text {eff }}$ is the effective anisotropy constant that mostly depends on the chemical composition of the particles and $\mathrm{V}$ the particle volume, $\omega$ is the angular frequency of the AC field, $n(D) d D$ is the number of particles per mass of sample with diameters in the $(D, D+d D)$ interval and $m(D)$ the magnetic moment of particles of diameter D. This mathematical expression is the responsible for the curves shown in Figs. $1 \mathrm{~h}$ and $1 \mathrm{k}$. The $\chi^{\prime \prime}(\mathrm{T})$ profile, as a hallmark of the particulate magnetic species, can be used to detect the different types of magnetic particles present in the biological tissue (Fig. 2). Hence, as a strightforward application, variations of the $\chi^{\prime \prime}(\mathrm{T})$ profile from sample to sample can be used to investigate metabolic processes associated to changes in the magnetic particle assembly ${ }^{23}$. Eventually, the analysis of $\chi^{\prime \prime}(\mathrm{T})$ consists in fact of a sort of magnetic spectroscopy in the temperature domain (differently from usual frequency domain analyses), whose data can be interpreted like a spectrum to quantify the different ironcontaining mineralised species present in the biological sample.
A numerical protocol to carry out this task is described in the next section.

\section{The quantification protocol}

\section{Magnetic properties of pure species: Standards}

Both the shape and the location in temperature of the $\chi^{\prime \prime}(\mathrm{T})$ profile are characteristic of every mineralised Fe-containing species. It does occur, e.g., for ferritin iron, synthetic nanoparticles for drug delivery, or contrast agents for MRI (Fig. $2 \mathrm{a})$. These $\chi^{\prime \prime}(\mathrm{T})$ profiles, previously obtained for the individual species, will be used as standards in the analysis of tissue samples.

In the preparation of the standards, however, it should be taken into account that, depending on the magnetic nature of the mineral particles and on their concentration, eventual dipolar interparticle interactions may alter their magnetic behaviour ${ }^{29}$. This issue is analysed below under the headings non-interacting regime and interacting regime.
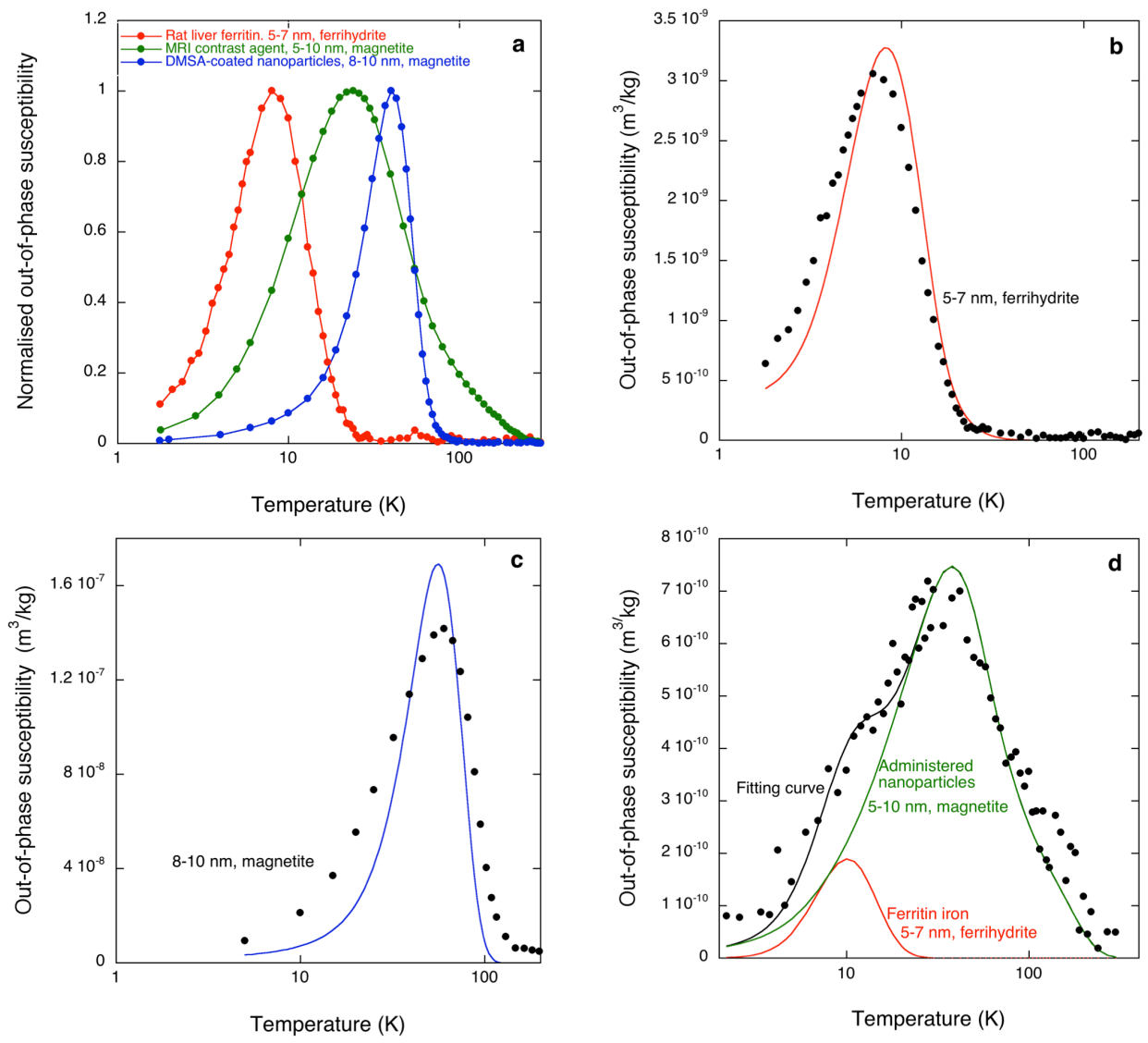

Fig. 2. Temperature dependence of the out-of-phase magnetic susceptibility of A) Mineral iron in the form of particles of different average sizes (from references ${ }^{23,25,}$ $\left.{ }^{43}\right)$, B) ferritin iron in a mouse liver with genetically induced iron overload ${ }^{44}$, C) magnetite nanoparticle carriers for drug delivery in a mouse liver ${ }^{24}$, D) magnetic nanoparticles from a superparamagnetic MRI contrast agent coexisting with ferritin iron in a rat liver ${ }^{23}$. In figure $A$ the solid line is just a guide for the eye; in figures $B$, $C$ and $D$, the solid lines represent the optimum fitting curve used in the particle quantification (see text). 
NON-INTERACTING REGIME. The iron-containing cores of ferritins constitute a good example of very small ironcontaining particles offering negligible interparticle dipolar interactions in biological tissues. In physiological conditions, ferritin is the main iron-containing mineral in the organism with reversible iron storage function ${ }^{45}$. This protein is formed by phosphorous rich ferrihydrite mineral particles of around 5-8 $\mathrm{nm}$ at the core of the protein shell. Due to the existence of this shell separating the cores, and to the predominantly antiferromagnetic nature of the iron-containing mineral, the interparticle dipolar interaction is very weak. With slight variations, depending on tissue and physiological conditions, the out-of-phase susceptibility maximum for ferritin is usually located, for a measuring frequency of $10 \mathrm{~Hz}$, at about $10 \mathrm{~K}$, a temperature substantially lower than for other nanoparticles in use (see Figs. 2a and 2d), and thus not interfering with the quantification of other species.

Within the non-interacting regime, also met by ferrimagnetic nanoparticles at very low concentrations, the magnetic quantity whose only contribution derives from the particles magnetism, $\chi^{\prime \prime}(\mathrm{T})$, results linearly proportional to the particles concentration (Fig. 2b).

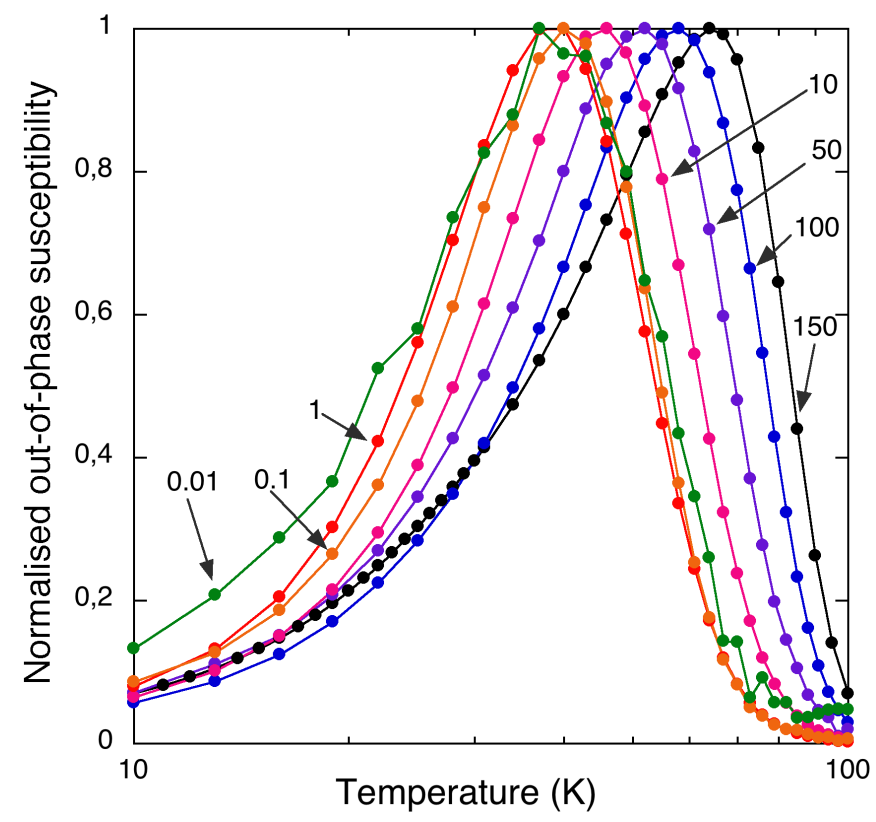

Fig. 3. Temperature dependence of the out-of-phase susceptibility of different agar-based nanoparticle dilutions. Although the out-of-phase susceptibility maximum per mass of particles is progressively depressed for increasing particle concentration (see text) the data are here shown normalised to their maximum. The numbers indicate the nominal nanoparticle concentration in milligrams of iron per gram of dry sample. For the lowest nanoparticle content the magnetic signal is the weakest, leading to the most intense scattering of the points due to experimental noise. Also for the three lower particle contents the data are rather coincident since the particles behave in the noninteracting (/dilute) regime.

INTERACTING REGIME. Magnetic suspensions for biomedical applications commonly contain ferrimagnetic (i.e., with a relatively high magnetic moment) iron oxide nanoparticles with a given size distribution that is intended to be as monodisperse as possible. In this case, should particle aggregation occur, or above a certain concentration (interacting regime), the particles magnetic moment dynamics is altered by interparticle dipoledipole interactions. More specifically, for a given particle size or particle size distribution, the increase of the degree of dipolar interaction, either due to particle aggregation or to a higher particle concentration, has two effects on the out-of-phase susceptibility per mass of particles: $i)$ the $\chi^{\prime \prime}(\mathrm{T})$ maximum shifts to higher temperatures (Fig. 3) and ii) its height decreases ${ }^{23}$. From this it appears obvious that the quantification process becomes invalidated if a standard that does not reasonably represent the interaction degree of the unknown sample is chosen.

However, if a given degree of particle aggregation takes place throughout the tissue, the height of the $\chi^{\prime \prime}(\mathrm{T})$ profile is expected to be virtually proportional to the concentration of the aggregates in the tissue ${ }^{23}$ (Figs. 2c and 2d). Therefore, it will be feasible to quantify aggregated particles by using standards with degrees of dipolar interactions comparable to those detected in the tissue under consideration.

\section{Management of the standards}

To model the interparticle interaction phenomenon, the particulate species under consideration can be dispersed in agar, a medium usually taken to simulate the tissue, using an ultrasonic bath to get a homogeneous distribution of the particles in the agar, at different particle concentrations. Then, to carry out the magnetic measurements, it results practical to freeze dry the gel making it possible to characterise a solid powder; this drying process does not alter significantly the magnetic behaviour.

After measuring the AC susceptibility of the so prepared standards, it is very practical to fit an analytical function $\chi_{i}{ }^{\prime \prime}(T)$, to the $\chi^{\prime \prime}(\mathrm{T})$ data points of every standard (the subscript $i$ represents the standard). This function, obtained to avoid the experimental noise of the standard raw data and being continuous to facilitate the subsequent calculation, can be in principle of any type, e.g. polynomial, but linear combinations of other smooth forms as gaussian or lognormal functions can also be used as far as they reasonably fit to the experimental points. In this aspect, we should remind that the $\chi^{\prime \prime}(\mathrm{T})$ profile, although assumed characteristic of a given nanoparticle assembly, depends in a rather complex way on many properties of the particles, therefore its functional form $\chi_{i}^{\prime \prime}(T)$ has to be based on phenomenological criteria.

If the tissue contains particles at low concentration, its $\chi^{\prime \prime}(T)$ profile will be nearly identical to that of the low particle concentrations standards (dilute regime). If the $\chi^{\prime \prime}(\mathrm{T})$ profile for the tissue appears at higher temperatures we may interpret that i) there is a magnetic influence of interparticle interactions from higher particle concentrations or from particle aggregation, or ii) the tissue contains larger (or chemically different) particles. To distinguish between these two possibilities, it becomes practical to see whether or not the preexponential factor $\tau_{0}$ (see its definition above), estimated for example by a method that just needs $\chi^{\prime}(T)$ and $\chi^{\prime \prime}(T)$ data at only one frequency ${ }^{23}$, is of the 
order of the expected value for the noninteracting regime. On the contrary, a $\chi^{\prime \prime}(\mathrm{T})$ profile appearing at temperatures lower than those for the dilute particle standard may not suggest a different particle arrangement in the tissue but actually a reduction of particle sizes most likely giving rise to iron release to the organism. In general, pharmacokinetic processes as particle clustering could be inferred by analyzing the dipoledipole interaction strength ${ }^{23,46,47}$.

\section{Obtention of the particulate content in the tissue}

With the above mentioned fitting functions $\chi_{i}^{\prime \prime}(T)$ in hand, it is possible to choose the function (i.e. the standard) that optimally represents the location in temperature of the $\chi^{\prime \prime}(\mathrm{T})$ data of the tissue. If the nanoparticles in the tissue are quite apart from each other (dilute regime) the low concentration standards will be a good representation; on the other hand, little particle aggregations or local higher concentrations will be manifested by a shift to higher temperatures of the $\chi^{\prime \prime}(\mathrm{T})$ profile and then other higher concentration standards will be a better choice. Once decided which is the $\chi_{i}^{\prime \prime}(\mathrm{T})$ profile of the standard that optimally represents the location in the temperature axis of the $\chi^{\prime \prime}(\mathrm{T})$ profile, the scaling factor $a$ that fits the experimental $\chi^{\prime \prime}(\mathrm{T})$ data can be obtained from

$$
\chi^{\prime \prime}(\mathrm{T}) \approx a \chi_{\mathrm{i}}{ }^{\prime}(\mathrm{T}) .
$$

This can be done through a least squares fitting algorithm, e.g. of a Levenberg-Marquardt type which is a built-in procedure in the most common data analysis computer applications. On doing so, the particle content in the tissue sample is obtained as $a$ times the particle content in the standard. Also should we expect contributions to $\chi^{\prime \prime}(\mathrm{T})$ coming from the presence of nanoparticles of neatly different nature, as in the case shown in Fig $2 \mathrm{~d}$, the same procedure can be followed in the form $\chi^{\prime \prime}(\mathrm{T}) \approx$ $\sum a_{\mathrm{i}} \chi_{\mathrm{i}}^{\prime \prime}(\mathrm{T})$, yielding the corresponding $a_{\mathrm{i}}$ values associated to the contents of each particulate species in the sample. We should indicate that the number of particles is not a good measure of the particle content since the contribution of one particle to $\chi^{\prime \prime}(\mathrm{T})$ is size-dependent. Instead, as the comparison between $\chi^{\prime \prime}(\mathrm{T})$ data of the tissue sample and the chosen standard is done by assuming identical size distributions, it results practical to express the particle content as particulate iron content. It should not be forgotten, in any case, that the number that actually matters (particle volume, particle surface, etc.) in particle quantification depends on the context of application.

\section{Application examples of the quantification protocol}

In this section we will outline previous results of nanoparticle quantification based on AC magnetic susceptibility data of tissue samples.

The biodistribution of superparamagnetic MRI contrast agents was studied by this method in a rat model. In this work the simultaneous detection and quantification of ferritin and the contrast agent in liver and spleen tissues was accomplished ${ }^{23}$. Furthermore, a different particle aggregation degree was found in the liver and the spleen tissues, indicating that at short times after the particle administration it is possible to distinguish an effect of the particle aggregation due to the tissue ultrastructure. In the frame of magnetic nanoparticles for drug delivery, we have analyzed the fate of dimercaptosuccinic acid-coated magnetic nanoparticles (DMSA-MNP) in mouse models. Ironbased magnetic nanoparticles usually accumulate in the liver and the spleen. By analyzing the out-of-phase susceptibility of several organs, including the heart, brain or kidneys, we found that in addition to the particle accumulation in liver and spleen tissues, there was a preferential accumulation of particles in the lung $^{48}$. This effect may be a consequence of the DMSA coating ${ }^{49}$. These results have opened the possible use of the DMSA-MNP as carriers for preferential lung targeting.

The main interest on DMSA-MNP for biomedical applications was to evaluate their role as carriers of interferon- $\gamma$ (IFN- $\gamma$ ), a citokyne used for cancer immunotherapy ${ }^{50}$. We found that there was a change on the biodistribution pattern when IFN- $\gamma$ was adsorbed to DMSA-MNP. In particular, a decrease of the accumulation of the particles in the lungs was observed ${ }^{51}$. This effect is probably due to changes on the particles surface charge and size induced by the cytokine.

Results from AC magnetic susceptibility measurements have also had a key role on the analysis of the effect of using an external magnet to target the particles to tumors. We were able to prove that the use of an external magnet resulted in an increase of the particles accumulation in the tumor tissues ${ }^{25,48}$. In addition to the particles coating or the use of external magnets, other factors that may alter the nanoparticles biodistribution such as the anesthetic used during the particle administration have been evaluated. Interestingly, we found that there is a clear effect on the number of particles that reaches the lungs when using two types of anesthetics ${ }^{52}$. In particular, the accumulation in the lungs was lower when using inhaled isoflurane than when using an intraperitoneally injected ketamine/xylazine anesthetic mixture. In contrast, we found that the number of particles in liver tissue was not affected by the anesthesia used.

AC magnetic susceptibility measurements have allowed us to study the particles transformations in tissues over periods up to three months after administration ${ }^{24}$. Two processes were observed in this study. First, a time decrease of the number of particles in each organ was found. In particular, the amount of particles found in the liver, spleen or lungs three months postadministration was significantly lower than the first week after nanoparticle injection. Second, transformation of the particles also occurred. A reduction in particle size, probably also accompanied by a disaggregation process reducing the dipolar interactions, was observed in these tissues. It is important to mention that it was still possible to observe particles in the liver, spleen and lungs of animals 90 days post-administration, indicating that experiments with longer monitoring times are required to see the complete metabolization of the particles. In this study, as the particles are transformed with time, it was only possible to do a qualitative analysis rather than a quantitative one, due to the lack of standards for the degraded 
particles within the tissue. This challenge, among some others, will be described in the following section.

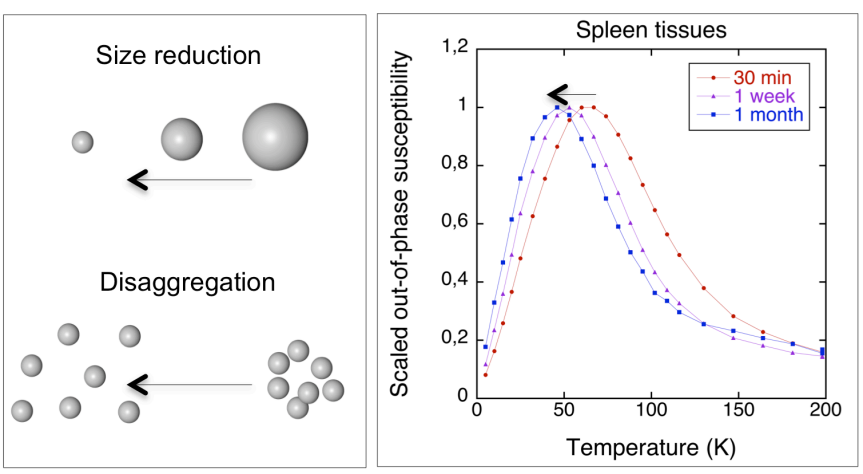

Fig. 4. Temperature dependence of the out-of-phase susceptibility, $\chi^{\prime \prime}(T)$, of spleen tissues from animals sacrificed at different times (30 minutes, 1 week and 1 month) after magnetic nanoparticles administration. The shift on the location of the susceptibility maxima towards lower temperatures over time is related either to a change in particles size, aggregation or both.

\section{Challenges of the quantification of magnetic nanoparticles by $A C$ magnetic susceptibility measurements}

\section{Detection limits}

For biomedical applications, the dosage of magnetic nanoparticles used is generally very low. This fact, together with a specific pattern of accumulation within the different organs may lead to undetectable nanoparticle contributions to the out-of-phase susceptibility. This has happened when characterizing brain, heart or kidney tissues of animals after the injection of DMSA-MNP ${ }^{25}$. However, intrinsic noise levels in the data can be used to estimate the detection limits of the technique. In the case of DMSA-MNP, they are of the order of $3 \mu \mathrm{g} \mathrm{Fe} / \mathrm{g}$ dry weight. Therefore, beyond a too simplistic analysis in terms of whether or not the particles have reached the tissue, a magnetic signal below the noise level indicates that the particulate iron content may be equal at most to the detection limit value.

\section{Particles degradation}

In long term studies the particles start to degrade in the tissues $^{15,16,20}$, leading to changes on the magnetic properties of the particles. This processes result in a non-straightforward analysis of the AC magnetic susceptibility data for quantitative purposes. The shift of the $\chi$ ”(T) maximum towards lower temperatures over time indicates nanoparticle disaggregation, reduction of nanoparticle size, or both (Fig. 4).

Nanoparticle quantification in tissues taken after the beginning of the degradation process would require a "library" of standards containing particles with different aggregation degree and size. Moreover, the use of a local technique as, for example, TEM will help on the selection of the most appropriated standard optimally matching the properties of the particles in the tissue.

\section{Synthesis of larger particles and particle size control}

As it has been mentioned before, because of its low toxicity and the existence of natural routes for its biodegradation, iron-based nanoparticles are preferred for biomedical applications ${ }^{53}$. In addition, for drug delivery, the optimal particle size ranges are between 10 to $100 \mathrm{~nm}$, that result in the longest blood circulation times ${ }^{4}$. Interestingly iron-based particles of these sizes present an out-of-phase magnetic susceptibility maximum that usually falls within the technically measurable temperature frame of the AC susceptometers, making this technique suitable for the particle characterization in biological samples. For the study of the accumulation of larger (micrometer size) particles in tissues, e.g. lung deposition of particles from aerosols ${ }^{54}$, the complete profile of the particles out-of-phase susceptibility may be partially or totally out of the available temperature window of the AC susceptibility measurements imposing a serious problem for quantification purposes. Narrow particle size distributions will facilitate the quantification protocol.

The best approach for a better control of the particles properties is known as 'bottom-up' synthesis ${ }^{55}$. This process generates larger nanostructures from single atoms, allowing a more homogeneous and narrower size and shape distribution. The most widely used methods to obtain magnetic nanoparticles are based on the precipitation of salts in aqueous medium to produce iron oxide nanoparticles. One option is to oxidize a ferrous hydroxide suspension by $\mathrm{H}_{2} \mathrm{O}_{2}$ or $\mathrm{KNO}_{3}$ obtaining particles larger than $20 \mathrm{~nm}$ in size ${ }^{56}$. Another option is the coprecipitation of stoichiometric mixtures of ferrous and ferric salts in basic medium. By this synthesis routes, large amounts of particles from 2 to $12 \mathrm{~nm}$ can be prepared ${ }^{57}$.

A different synthesis route, based on the thermal decomposition of metallic compounds in organic solvents and containing stabilizing surfactants, is able to produce magnetic nanoparticles with good crystallinity and highly monodisperse but usually hydrophobic ${ }^{58}$. The major drawback from this route is that, in biomedical applications, there is a necessary step after the synthesis to stabilize the particles in aqueous medium by modification or substitution of the surfactant coating.

Finally, biomimetics offers the possibility of using lowtemperature pathways for the production of a variety of structure-specific magnetic nanocrystals with sizes and shapes not readily obtained via conventional inorganic chemical techniques. The self-assembly and directed assembly of synthetic polymers, viruses, peptides, DNA molecules, proteins and various polymer-based hybrid materials acting as matrices, scaffolds and templating agents, permit molecular-level control over the produced material ${ }^{59}$. The resulting magnetosomes consist of two parts: an organic layer and an inorganic magnetic nanocrystal, usually $35-120 \mathrm{~nm}$ in size, structurally perfect and single domain with narrow size distribution.

Either the precipitation of salts in aqueous medium, the thermal decomposition of metallic compounds or the magnetosomes match the requirements for biomedical applications. Nevertheless, there is still room for improvements in terms of narrower size distributions, improved crystallinity and 
homogeneity in chemical composition, all of these aspects strongly related to the particles magnetic properties. It will also be desirable to develop cleaner synthetic routes able to preserve the colloidal and magnetic properties of the particles.

\section{Conclusions}

AC magnetic susceptibility measurements of biological samples are an alternative tool for the quantitative determination of magnetic nanoparticles. One of the hallmarks of this technique is that it allows detecting changes of particle sizes, being especially useful to get new insights on particle biotransformations over time. Furthermore, this technique allows the distinction of tissue endogenous iron-containing species from magnetic nanoparticles. To improve the performance of the magnetic nanoparticles in biomedicine homogeneous magnetic nanoparticles should be used.

\section{Acknowledgements}

LG is the Beneficiary of a post-doctoral grant from the AXA Research Fund. This work was partially supported by grants from the Spanish Ministry of Economy and Competitiveness (MAT2011-23641), the Madrid regional government (S009/MAT-1726), Junta de Andalucía (P11-FQM-7074), Carlos III Health Institute (PI060549) and the EU-FP7 MULTIFUN project (NMP-Large Ref. 246479). Authors would like to acknowledge the use of Servicio General de Apoyo a la Investigación-SAI, Universidad de Zaragoza.

\section{Notes and references}

${ }^{a}$ Department of Biomaterials and Bioinspired Materials, Instituto de Ciencia de Materiales de Madrid (ICMM)/CSIC, Sor Juana Inés de la Cruz 3, Cantoblanco, 28049 Madrid, Spain.

$b$ Department of Materials and Fluids Science and Technology, Universidad de Zaragoza, María de Luna 3, 50018 Zaragoza, Spain.

$\dagger$ Quantum Design, Inc., http://www.qdusa.com.

1. R. N. Kostoff, J. A. Stump, D. Johnson, J. S. Murday, C. G. Lau and W. M. Tolles, J. Nanopart. Res., 2006, 8, 301-321.

2. J.-f. Liu, Z.-s. Zhao and G.-b. Jiang, Environ. Sci. Technol., 2008, 42, 6949-6954.

3. M. K. Jones and J. Balen, PLoS Negl. Trop. Dis., 2007, 1, e159.

4. M. Colombo, S. Carregal-Romero, M. F. Casula, L. Gutiérrez, M. P. Morales, I. B. Böhm, J. T. Heverhagen, D. Prosperi and W. J. Parak, Chem. Soc. Rev., 2012, 41, 4306-4334.

5. Q. Pankhurst, N. Thanh, S. Jones and J. Dobson, J. Phys. D: Appl. Phys., 2009, 42, 224001.

6. A. G. Roca, R. Costo, A. F. Rebolledo, S. Veintemillas-Verdaguer, P. Tartaj, T. González-Carreño, M. P. Morales and C. J. Serna, J. Phys. D: Appl. Phys., 2009, 42.

7. W. R. Sanhai, J. H. Sakamoto, R. Canady and M. Ferrari, Nat. Nanotechnol., 2008, 3, 242-244.
8. S. J. H. Soenen and M. De Cuyper, Nanomedicine, 2010, 5, 12611275.

9. E. Casals, E. Gonzalez and V. Puntes, J. Phys. D: Appl. Phys., 2012, 45, 443001 .

10. K. Thomas, P. Aguar, H. Kawasaki, J. Morris, J. Nakanishi and N. Savage, Toxicol. Sci., 2006, 92, 23-32.

11. M. Lévy, C. Wilhelm, M. Devaud, P. Levitz and F. Gazeau, Contrast Media Mol. Imaging, 2012, 7, 373-383.

12. B. Chertok, B. A. Moffat, A. E. David, F. Yu, C. Bergemann, B. D. Ross and V. C. Yang, Biomaterials, 2008, 29, 487-496.

13. A. Al Faraj, F. Gazeau, C. Wilhelm, C. Devue, C. L. Guérin, C. Péchoux, V. Paradis, O. Clément, C. M. Boulanger and P.-E. Rautou, Radiology, 2012, 263, 169-178.

14. H. Hong, Y. Zhang, J. Sun and W. Cai, Nano Today, 2009, 4, 399413.

15. J. D. López-Castro, A. V. Maraloiu, J. Delgado, J. Calvino, M.-G. Blanchin, N. Gálvez and J. M. Domínguez-Vera, Nanoscale, 2011, 3, 4597-4599.

16. L. Lartigue, D. Alloyeau, J. Kolosnjaj-Tabi, Y. Javed, P. Guardia, A. Riedinger, C. Pechoux, T. Pellegrino, C. Wilhelm and F. Gazeau, ACS Nano, 2013.

17. A. Brown and N. Hondow, in Frontiers of Nanoscience, ed. S. Huw, Elsevier, 2013, vol. Volume 5, pp. 95-120.

18. L. F. Gamarra, Int. J. Nanomed., 2010, 5, 203.

19. J. Oh, M. D. Feldman, J. Kim, C. Condit, S. Emelianov and T. E. Milner, Nanotechnology, 2006, 17, 4183.

20. M. Levy, N. Luciani, D. Alloyeau, D. Elgrabli, V. Deveaux, C. Pechoux, S. Chat, G. Wang, N. Vats, F. Gendron, C. Factor, S. Lotersztajn, A. Luciani, C. Wilhelm and F. Gazeau, Biomaterials, 2011, 32, 3988-3999.

21. C. Knopke, F. Wiekhorst, D. Eberbeck, I. Gemeinhardt, M. Ebert, J. Schnorr, S. Wagner, M. Taupitz and L. Trahms, IEEE Trans. Magn., 2013, 49, 421-424.

22. J. B. Weaver, X. Zhang, E. Kuehlert, S. Toraya-Brown, D. B. Reeves, I. M. Perreard and S. Fiering, Nanotechnology, 2013, 24, 325502.

23. A. López, L. Gutiérrez and F. J. Lázaro, Phys. Med. Biol., 2007, 52, 5043-5056.

24. R. Mejías, L. Gutiérrez, G. Salas, S. Pérez-Yagüe, T. M. Zotes, F. J. Lázaro, M. P. Morales and D. F. Barber, J. Controlled Release, 2013, 171, 225-233.

25. L. Gutiérrez, R. Mejías, D. F. Barber, S. Veintemillas-Verdaguer, C. J. Serna, F. J. Lázaro and M. P. Morales, J. Phys. D: Appl. Phys., 2011, 44, 255002.

26. J. Dormann, L. Bessais and D. Fiorani, Journal of Physics C: Solid State Physics, 1988, 21, 2015.

27. J. Garcia-Palacios, Adv. Chem. Phys., 2000, 112, 1-210.

28. R. Egli, Geophys. J. Int., 2009, 177, 395-420.

29. M. F. Hansen, P. Jönsson, P. Nordblad and P. Svedlindh, J. Phys.: Condens. Matter, 2002, 14, 4901.

30. Y. L. Raikher and V. Stepanov, J. Exp. Theor. Phys., 2008, 107, 435444.

31. L. Corá, F. Romeiro, M. Stelzer, M. Américo, R. Oliveira, O. Baffa and J. Miranda, Adv. Drug Delivery Rev., 2005, 57, 1223-1241.

32. W. Weitschies, O. Kosch, H. Mönnikes and L. Trahms, Adv. Drug Delivery Rev., 2005, 57, 1210-1222. 
33. O. Girard, R. Ramirez, S. McCarty and R. Mattrey, Contrast Media Mol. Imaging, 2012, 7, 411-417.

34. A. Ruiz, Y. Hernández, C. Cabal, E. González, S. VeintemillasVerdaguer, E. Martínez and M. P. Morales, Nanoscale, 2013.

35. L. Pauling, Proc. Natl. Acad. Sci. U. S. A., 1977, 74, 2612-2613.

36. D. J. Craik, Magnetism: Principles and Applications, by Derek J. Craik, pp. 468. ISBN 0-471-95417-9. Wiley-VCH, September 2003., 2003, 1.

37. S. Kilcoyne and R. Cywinski, J. Magn. Magn. Mater., 1995, 140, 1466-1467.

38. L. Gutiérrez and F. J. Lázaro, J. Magn. Magn. Mater., 2007, 316, 136-139.

39. Y.-X. J. Wang, S. M. Hussain and G. P. Krestin, Eur. Radiol., 2001, 11, 2319-2331.

40. C. Sun, J. S. Lee and M. Zhang, Adv. Drug Delivery Rev., 2008, 60, 1252-1265.

41. A. Jordan, R. Scholz, P. Wust, H. Fähling and R. Felix, J. Magn. Magn. Mater., 1999, 201, 413-419.

42. M. Shliomis and V. Stepanov, Adv. Chem. Phys., 1994, 87, 1-30.

43. L. A. Cohen, L. Gutierrez, A. Weiss, Y. Leichtmann-Bardoogo, D. L. Zhang, D. R. Crooks, R. Sougrat, A. Morgenstern, B. Galy, M. W. Hentze, F. J. Lazaro, T. A. Rouault and E. G. MeyronHoltz, Blood, 2010, 116, 1574-1584.

44. L. Gutiérrez, C. Quintana, C. Patiño, J. Bueno, H. Coppin, M. P. Roth and F. J. Lázaro, BBA - Mol. Bas. Dis., 2009, 1792, 541-547.

45. P. M. Harrison and P. Arosio, Biochim. Biophys. Acta, Bioenerg., 1996, 1275, 161-203.

46. M. Lévy, F. Gazeau, J.-C. Bacri, C. Wilhelm and M. Devaud, Phys. Rev. B: Condens. Matter, 2011, 84, 075480.

47. M. Levy, C. Wilhelm, N. Luciani, V. Deveaux, F. Gendron, A. Luciani, M. Devaud and F. Gazeau, Nanoscale, 2011, 3, 44024410.

48. R. Mejías, S. Pérez-Yagüe, L. Gutiérrez, L. I. Cabrera, R. Spada, P. Acedo, C. J. Serna, F. J. Lázaro, T. Villanueva, M. D. P. Morales and D. F. Barber, Biomaterials, 2011, 32, 2938-2952.

49. S. B. Chaves, L. M. Lacava, Z. G. M. Lacava, O. Silva, F. Pelegrini, N. Buske, C. Gansau, P. C. Morais and R. B. Azevedo, IEEE Trans. Magn., 2002, 38, 3231-3233.

50. M. J. Smyth, D. I. Godfrey and J. A. Trapani, Nat. Immunol., 2001, 2, 293-299.

51. L. Gutiérrez, R. Mejías, D. Barber, S. Veintemillas-Verdaguer, C. Serna, F. Lázaro and M. Morales, J. Phys. D: Appl. Phys., 2011, 44, 255002.

52. L. Gutierrez, R. Mejias, F. J. Lazaro, C. J. Serna, D. F. Barber and M. P. Morales, IEEE Trans. Magn., 2013, 49, 398-401.

53. P. Tartaj, M. P. Morales, T. Gonzalez-Carreño, S. VeintemillasVerdaguer and C. J. Serna, Adv. Mater., 2011, 23, 5243-5249.

54. G. R. Zosky, C. E. Boylen, R. S. Wong, M. N. Smirk, L. Gutiérrez, R. C. Woodward, W. Shan Siah, B. Devine, F. Maley and A. Cook, Respirology, 2013, DOI: 10.1111/resp.12150.

55. G. Salas, R. Costo and M. d. P. Morales, in Frontiers of Nanoscience, eds. M. d. 1. F. Jesus and V. Grazu, Elsevier, 2012, vol. Volume 4, pp. 35-79.

56. M. Marciello, V. Connord, S. Veintemillas-Verdaguer, M. A. Verges, J. Carrey, M. Respaud, C. J. Serna and M. P. Morales, J. Mater. Chem., 2013, 1, 5995-6004.
57. Y. Luengo, S. Nardecchia, M. P. Morales and M. C. Serrano, Nanoscale, 2013.

58. G. Salas, C. Casado, F. J. Teran, R. Miranda, C. J. Serna and M. P. Morales, J. Mater. Chem., 2012, 22, 21065-21075.

59. T. Prozorov, D. A. Bazylinski, S. K. Mallapragada and R. Prozorov, Mater. Sci. Eng., R, 2013, 74, 133-172. 